\title{
Meganoster \\ Implementing a culture of creativity Pop-up making spaces and participating events in academic libraries
}

$\mathbf{I}_{\mathrm{b}}^{\mathrm{n}}$ the 21st century, the academic library can be seen as laboratory or making space that can inspire and stimulate creativity within the scholarly community. As many academic libraries are feeling the economic crunch and constant challenge of having to prove their value, I believe that participatory activities engage patrons in new ways that can help the libraries further their impact within their communities. Making spaces are fun, can be affordable, and provide an opportunity for library employees to engage with each other, as well as with patrons. Making activities get patrons in the library doors but can also encourage partnerships and cross-disciplinary collaboration across campuses.

This article will discuss the importance of making events, cross-disciplinary collaboration, and using outreach and marketing skills to engage and educate one's community. Participatory programming is a means of looking outward and engaging patrons in a way that allows them to be creators and have their voices heard.

\section{Making in academic libraries}

At the ACRL 2013 conference in Indianapolis, Indiana, Char Booth, Lia Friedman, Adrienne Lai, and Alice Whiteside spoke about the importance of building goodwill in the library from the inside out. ${ }^{1}$ All of the projects presented on the panel were innovative, and many of them included a making aspect. Examples of events included in this presentation were button making at Claremont College, DIY photobooths at University of California-San Diego, My \#HUNTLIBRARY at North Carolina State University, and re:book 2013 at Claremont College. ${ }^{2}$

On November 19, 2013, Theresa R. McDevitt posed a question to the ARLIS/NA electronic list asking for advice on how to help students use the library to de-stress during finals with activities that are cheap, easy, and non-messy. Some of the making events suggestions included jigsaw puzzles, graffiti walls, baskets of yarn and knitting needles, coloring, and origami.

Some additional making events that are happening in academic libraries include pumpkin decorating and snowflake making at Rutgers University Libraries (RUL); making bookmarks at Carl Sandburg College; confess your stress selfies as therapy photo booth at Virginia Tech; box making at Southern Illinois UniversityCarbondale (SIUC); and edible books festivals happening at many academic libraries in the United States. The events described above can be referred to as participatory events, which essentially are pop-up making spaces, similar to a makerspace.

Makerspaces can cost a fair amount of money to create, be space-consuming, and need monitoring and sustainability funding. Pop-up making spaces can be easily put up, taken down, sent from one library to another, and they use little space for storing materials.

Implementing a climate of creativity with participatory activities within one's library can help patrons build good problem-solving skills and become lifelong learners and supporters of the library. Libraries can use making events as a means to spread the importance of learning and the power that libraries bring to the scholarly process. When one is having fun, one is more likely to learn and support the organization that is providing the ephemeral experience. As Erin Fisher states, "making provides opportunities

Megan Lotts is arts librarian at Rutgers, the State University of New Jersey, email: megan.lotts@rutgers. edu

(0) 2015 Megan Lotts 
for people to learn with their hands" and those are skills that are applicable when conducting scholarly research in an academic library. ${ }^{3}$

The next section of this article will discuss three making/participatory events coordinated by the author at RUL that will provide insight and tips in creating your own making spaces. The events included are edible books, holiday card making, and polynomiography. These events created a positive disruption within the library and can be a way of using outreach, engagement, and making as a means to connect with one's community as well as spread the importance of the role the library plays in the academic sphere.

\section{Edible books}

Many libraries have hosted edible books festivals since Judith A. Hoffberg and Béatrice Coron held the first festival in 1999. ${ }^{4}$ In planning a successful edible books festival there are five mains tasks that need to be addressed: budget, judges, prizes, volunteers for day of event, and good publicity. But the most important issue that always rises to the top is, Will there be enough participants?

Acquiring a budget for edible books is dependent on many factors. The event can be considered successful with a budget of as much as $\$ 250$ or as little as $\$ 100$, with a little creativity: look for local sponsors to donate prizes, ask the library to support food and drink, use library printing services for forms for the day of event and any other printing, and use community news and electronic list blasts to support greenfriendly, no-cost publicity.

Finding prizes, particularly if you are working with a smaller budget, can be timeconsuming. However, many participants don't enter an edible books festival for the prizes but more for the glory that comes with winning Best in Show. The local university bookstore on the Rutgers University (RU)-New Brunswick campus provides the grand prize every year of an e-reader.

When it comes to judges, it's important to find good cross-disciplinary partners. At both RU and SIUC, head chefs from Dining Services were invited to participate as judges. Having the right judges for the event not only gives a wide perspective of "taste," as in aesthetics, but also can encourage future projects and crossdisciplinary collaboration. Having administrators who play an important role on campus as judges reminds them of the innovative and exciting work being done in libraries.

It's important to have volunteers at any public or making event. Volunteers not only support 
was a conversation about the past, present, and future of the book, as well as how one might actually catalog an edible book.

\section{Holiday card making}

The RU Kilmer Library holiday card-making space was implemented during finals week in the fall of 2013. This event gave participants the opportunity to make and mail a card to anyone of their choosing, as well as enjoy free coffee, cookies, and candy. This project was intended to be a low-budget making event that could be mobile and easily re-created at any one of the 27 RU libraries and centers.

To obtain the supplies for this project, roughly three weeks prior to the event, an email was sent to all the RUL faculty and staff asking for donations of crafting supplies, including adhesives, colored paper, stickers, wrapping paper, markers, colored pens, recycled magazines, recycled calendars, beads, and buttons. Within a week of sending the email, I had enough crafting supplies to host card making and other participatory crafting events on a regular basis.

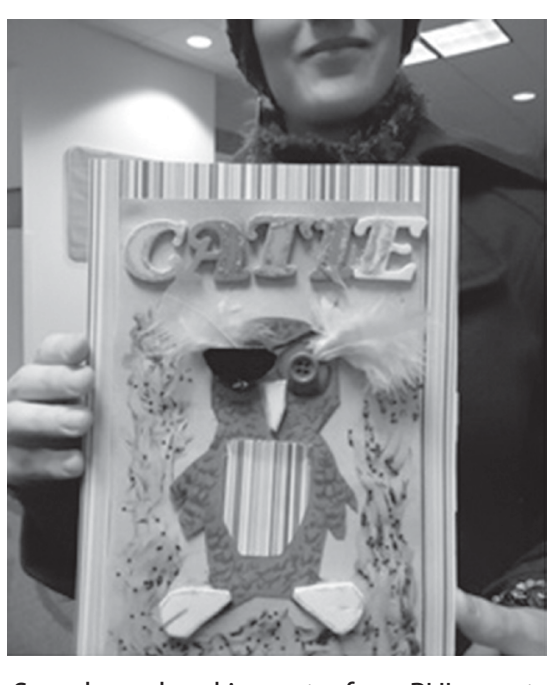

Sample card-making entry from RUL event. nomiography is also a digital

For the event, RUL purchased $\$ 40$ worth of stamps, $\$ 60$ worth of candy and cookies, and used the library coffee pots to provide free coffee. (It's best to buy fewer stamps and invest in more cookies.)

For the publicity aspect of the holiday card making space, the main focus was a digital campaign, including our regular campus partners who publicize our events on their Twitter feeds, Facebook pages, RUL website and social media, as well as all the RUL e-displays.

Volunteers were recruited for this event via the RUL electronic list to help pass out candy, coffee, and to engage with the participants. Most of the volunteers made a new friend and learned more about the needs of our students, faculty, and staff during the stressful time of finals.
Overall the students, faculty, and staff were thrilled with the opportunity to make and mail cards. Students were also delighted with free candy and coffee to get them through the stress of finals. The event caused a positive commotion in the library and also inspired students, faculty, and staff to take a break. This event also engaged participants and users of the library in a greater discussion about communication and how we currently engage or communicate within our own communities.

For Rutgers Day, April 28, 2013, and for Scarlet

\section{Polynomiography}

Knight Days, September 17, 2014, RUL hosted polynomial making events at the Art Library and the Library of Science and Medicine. At both events we used Bahman Kalantari's (from the RU Department of Computer Sciences) polynomigraphy software. Kalantari describes polynomiography as "algorithmic visualizations of one of the most basic and fundamental tasks in science and math: solving a polynomial equation. Polymedium that can be used to encourage creativity, artistry and discovery, with tremendous appeal for playful learning." 5

There was no fiscal cost to host the polynomiography events at RUL, with the exception of some "in kind" printing, library employee time to prepare for the event, and $\$ 60$ to purchase cookies. Free coffee was served, and the library coffee pots were used to make the coffee. Prior to both events, library computers were temporarily reserved, and the software was loaded onto the computers. Volunteers were recruited to help with the event from RUL and the RU Department of Computer Sciences.

At both events, once an image was created it could be sent to an email of your choosing or printed out on one of the libraries color printers at no cost to the participant. 
I created all of the publicity and circulated it digitally to keep fiscal costs down. One largescale poster was printed, in kind, by RUL SCC and placed outside the events to entice visitors into the library to use the software.

Overall this project was successful at highlighting the intersection of the Arts, Science, and Mathematics and showcasing the work of Kalantari. Both events gave individuals the opportunity to physically create a digital polynomilagraphy that they could share with others. Many participants left asking if the software was available for download, and a few K-12 math teachers approached Kalantari about the possibility of having his software integrated into their classrooms.

\section{Suggestions and tips}

When planning outreach events with a participatory aspect, it's important to make sure you know your audience. Find collaborators and partners outside of the libraries who share your interests and can help build your audience. Good partnerships can often produce fiscal support for events.

Make sure to get the word out. Create publicity email lists that you can

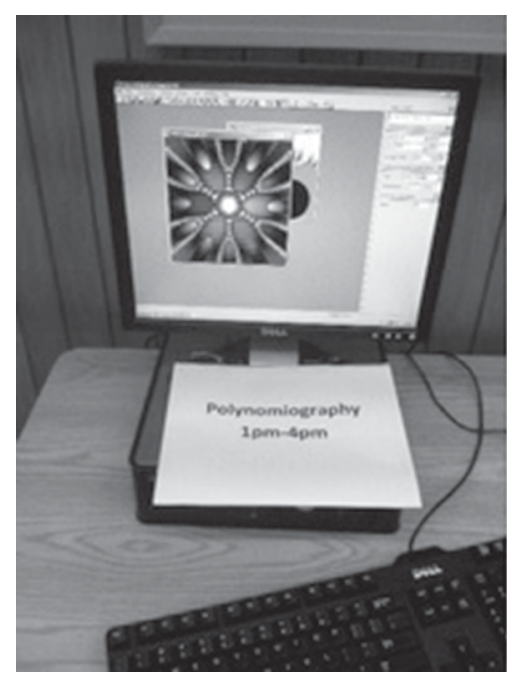

Polynomiography-making event. hands do the thinking, while they learn about the possibilities in the library.

The next step is to explore more participatory events in academic libraries that encourage making within one's community. In the fall of 2014, the RU Art Library implemented a Lego playing station that provides students, faculty, staff, and community members the opportunity to think with their hands using the medium of Legos. This project includes a crowdsourced collection of images of Lego models made in the Art Library and will be incorporated into the curriculum of the Rutgers Landscape Architecture Program and the Rutgers School of Arts \& Sciences Honors Colloquium. continue to update and use for each event. Make friends with the media departments on campuses. They know where the students are, what they pay attention to, and are always looking for interesting stories and happenings on college campuses. If your library has a communications director or marketing team, often these individuals and groups can be helpful in promoting your events.

As seen from the three events above, popup making spaces can be fun, affordable, and relatively easy to coordinate. Making events can provide the libraries an opportunity to further engage with patrons and also build goodwill among the library staff. But, more important, they get patrons in the doors and give them the opportunity to take a break and let their

\section{Conclusion}

Participatory and making events can bring positive attention to the library and invite patrons to share their skills and talents. Creating a positive disruption can bring community together and inspire individuals to become creators and good problem solvers. The library has the potential to be the leader in showcasing dynamic scholarly research that is being created on college campuses. Collaborating with students, faculty members, and departments brings people together and can open up a multi-layer conversation that allows the exploration of ideas from many different perspectives.

\section{Notes}

1. Char Booth, Lia Friedman, Adrienne Lai, and Alice Whiteside, "Love your library: building goodwill from the inside out and the outside in," SlideShare, www.slideshare.net/charbooth/ love-your-library-building-goodwill-from-theinside-out-and-the-outside-in.

2. Ibid.

3. Erin Fisher, "Makerspaces Move into Academic Libraries," http://acrl. ala. org/techconnect (2012).

4. Books2eat, www.books2eat.com/.

5. Bahman Kalantari, www.polynomiography. $\mathrm{com} / \mathrm{n}$ 